\title{
Generic Density and Small Span Theorem
}

\author{
Philippe Moser \\ Department of Computer Science, National University of Ireland, Maynooth Co. \\ Kildare, Ireland.
}

\begin{abstract}
We refine the genericity concept of Ambos-Spies, by assigning a real number in $[0,1]$ to every generic set, called its generic density. We construct sets of generic density any E-computable real in $[0,1]$, and show a relationship between generic density and Lutz resource bounded dimension. We also introduce strong generic density, and show that it is related to packing dimension. We show that all four notions are different. We show that whereas dimension notions depend on the underlying probability measure, generic density does not, which implies that every dimension result proved by generic density arguments, simultaneously holds under any (biased coin based) probability measure. We prove such a result: we improve the small span theorem of Juedes and Lutz, to the packing dimension setting, for $k$-bounded-truthtable reductions, under any (biased coin) probability measure.
\end{abstract}

Key words: Genericity, resource-bounded dimension, small span theorem

\section{Introduction}

Resource-bounded genericity [1] yields a randomness concept for the class $\mathrm{E}$ which interacts nicely with resource-bounded measure [16]. Informally speaking, generic sets are sets which cannot be predicted correctly infinitely often. Genericity has been used for the investigation of structural properties of NP (under appropriate assumptions) and E, see [2] for a survey; and yielded an improved version of the small span theorem of [12], to a stronger reduction notion [4], based on the relationship between measure and genericity.

Resource-bounded measure has recently been refined via effective dimension which is an effectivization of Hausdorff dimension, yielding applications in a variety of topics, including algorithmic information theory, computational

Email address: pmoser(at)cs.nuim.ie (Philippe Moser). 
complexity, prediction, and data compression $[18,14,19,7,5,10]$. Hausdorff dimension is a refinement of measure theory, where every measure zero class of languages is assigned a dimension, which is a real number between 0 and 1 . Another widely used dimension concept in fractal geometry, known as packing dimension (or strong dimension), was effectivized in [5]. A simple characterization of strong dimension via martingales has been given in [5], where the martingales' capital is required to grow unbounded and is not allowed to decrease too much after a certain number of rounds.

In this paper we connect genericity to resource-bounded dimension by introducing a quantified version of genericity, which is a refinement of genericity, as resource-bounded dimension is a refinement of resource-bounded measure. The idea is that every generic set is assigned a real number between 0 and 1 , called its generic density, and which corresponds to the density such a set cannot be predicted with. We construct sets of generic density any E-computable real $s \in[0,1]$. Similarly to resource-bounded strong dimension [5], we also introduce strong generic density. We show that strong generic density is related to strong dimension [5], in the sense that sets with a certain amount of randomness relatively to strong dimension, keep that amount of unpredictability relatively to strong generic density.

Next we show that all these four concepts, i.e. dimension, strong dimension, generic density and strong generic density, are indeed different.

All notions exposed so far are implicitly considered within the Cantor space of all languages under the uniform probability measure. This corresponds to the random experiment in which every membership bit of a language $L$ is chosen according to the toss of a fair coin. Probability measures other than the uniform probability measure occur naturally in applications, and the corresponding gale notion (resp. dimension notion) has been investigated in $[19,5]$ (resp. [9]). In section 6, we highlight a main difference between generic density and resource-bounded dimension, that is whereas the latter notion is dependent on the underlying probability measure, generic density is not; a similar result for genericity vs resource-bounded measure was given in [13]. More precisely we show that if the coin in the above random experiment is biased, then for two different biases the corresponding dimension notions differ, whereas the generic density notion remains the same. This outlines a nice feature of the generic density method over martingale based dimension: proofs obtained by generic density arguments are in some sense more informative, because all dimension results proved by generic density methods (i.e. showing some class contains some $s$-generic set) simultaneously hold in a wide variety of probability measure spaces. Such an example is given in the last section of this paper, where a small span theorem under any biased coin based probability measure is proved. 
More precisely we prove a small span theorem in the strong dimension setting, for $k$-bounded-truth-table reductions ( $k$-tt-reductions are a special case of Turing reductions, where only $k$ non-adaptive queries are allowed) under any biased coin based probability measure. The small span theorem [12] asserts that for every language $L$ in $\mathrm{E}$, either the set of languages reducible to $L$, called the lower span, or the set of languages to which $L$ reduces, called the upper span, has E-measure zero. The question whether the small span theorem still holds in the resource-bounded dimension setting - i.e. can Emeasure zero be replaced by E-dimension zero - was partially disproved in [3], where E-languages with both lower and upper span of E-dimension one were constructed. Nevertheless the small span theorem under polynomial many-one reductions holds for scaled dimension [11] and partially holds in the dimension setting as shown in [11], i.e. either the lower span has E-dimension zero or the upper span has E-measure zero. By adapting the proof of Ambos-Spies, Nies and Terwijn [4] combined with generic frequency arguments, we prove a small span theorem in the strong dimension setting for $k$-bounded truth table reductions, under any (biased coin) probability measure, i.e. we show that for any $L$ in $\mathrm{E}$, either the lower span (under $k$-tt-reductions) has $\mathrm{E}$ - $\vec{\beta}$-strong dimension zero (where $\vec{\beta}$ denotes the sequence of biases), or the upper span has E- $\vec{\beta}$-measure zero. $k$-bounded-truth-table reductions and $n^{\alpha}$-tt reductions $(\alpha<1)$ were considered in $[4,6]$, but only in the resource-bounded measure setting.

A preliminary version of this paper was published in [20].

\section{Preliminaries}

Let us fix some notations for strings and languages. A string is an element of $\{0,1\}^{n}$ for some integer $n$. For a string $x$, its length is denoted by $|x|$. $s_{0}, s_{1}, s_{2} \ldots$ denotes the standard enumeration of the strings in $\{0,1\}^{*}$ in lexicographical order, where $s_{0}=\lambda$ denotes the empty string. We sometimes enumerate the strings of size $n$ by $s_{0}^{n}, s_{2}^{n}, s_{2^{n}-1}^{n}$. Note that $|w|=2^{O\left(\left|s_{|w|}\right|\right)}$. If $x, y$ are strings, we write $x \leq y$ if $|x|<|y|$ or $|x|=|y|$ and $x$ precedes $y$ in alphabetical order. A sequence is an element of $\{0,1\}^{\mathbb{N}}$. If $w$ is a string or a sequence and $1 \leq i \leq|w|$ then $w[i]$ and $w\left[s_{i}\right]$ denotes the $i$ th bit of $w$. Similarly $w[i \ldots j]$ and $w\left[s_{i} \ldots s_{j}\right]$ denote the $i$ th through $j$ th bits.

For two strings $x, y$, the concatenation of $x$ and $y$ is denoted $x y$. If $x$ is a string and $y$ is a string or a sequence extending $x$ i.e. $y=x u$, where $u$ is a string or a sequence, we write $x \sqsubseteq y$. We write $x \sqsubset y$ if $x \sqsubseteq y$ and $x \neq y$.

A language is a set of strings. A class is a set of languages. The cardinal of a language $L$ is denoted $|L|$. Let $n$ be any integer. We identify language $L$ with 
its characteristic function $\chi_{L}$, where $\chi_{L}$ is the sequence such that $\chi_{L}[i]=1$ iff $s_{i} \in L$. Thus a language can be seen as a sequence in $\{0,1\}^{\mathbb{N}}$. We denote by $\mathrm{C}$ the Cantor space of all infinite binary sequences. $L 1 s_{n}$ denotes the initial segment of $L$ up to $s_{n-1}$ given by $L\left[s_{0} \cdots s_{n-1}\right]$, whereas $L \uparrow s_{n}$ denotes $L\left[s_{0} \cdots s_{n}\right]$.

We consider bounded truth-table reductions, here is a definition. Let $k \in$ $\mathbb{N}_{+}$. We say language $A$ is $k$-truth-table reducible to language $B$, denoted $A \leq_{k-t t}^{p} B$ if there exists a family of polynomial computable functions $f$ : $\{0,1\}^{*} \times\{0,1\}^{k} \rightarrow\{0,1\}$ (the evaluator) and $g_{i}:\{0,1\}^{*} \rightarrow\{0,1\}^{*}(1 \leq i \leq k$, the queries), such that for every string $x: A(x)=f\left(x, B\left(g_{1}(x)\right), \cdots, B\left(g_{k}(x)\right)\right)$. Such a reduction is denoted $f\left(g_{1}, \cdots, g_{k}\right)$. $A$ is bounded truth-table reducible to $B$ if it is $k$-truth-table reducible to $B$ for some $k$.

For a reducibility notion $r$, the lower span (resp. upper span) of a language $A$, denoted $A^{\geq_{r}}$ (resp. $A^{\leq_{r}}$ ) is the set of languages $B$ such that $B \leq_{r} A$ (resp. $\left.A \leq_{r} B\right)$.

\subsection{Lutz Resource-bounded Measure}

Lutz measure on $E$ [16] is obtained by imposing appropriate resource-bounds on a game theoretical characterization of classical Lebesgue measure, via martingales. A martingale is a function $d:\{0,1\}^{*} \rightarrow \mathbb{R}_{+}$such that, for every $w \in\{0,1\}^{*}, d(w)=(d(w 0)+d(w 1)) / 2$. This definition can be motivated by the following betting game in which a gambler puts bets on the successive membership bits of a hidden language $A$. The game proceeds in infinitely many rounds where at the end of round $n$, it is revealed to the gambler whether $s_{n} \in A$ or not. The game starts with capital 1 . Then, in round $n$, depending on the first $n-1$ outcomes $w=\chi_{A}[0 \ldots n-1]$, the gambler bets a certain fraction $\epsilon_{w} d(w)$ of his current capital $d(w)$, that the $n$th word $s_{n} \in A$, and bets the remaining capital $\left(1-\epsilon_{w}\right) d(w)$ on the complementary event $s_{n} \notin A$. The game is fair, i.e. the amount put on the correct event is doubled, the one put on the wrong guess is lost. The value of $d(w)$, where $w=\chi_{A}[0 \ldots n]$ equals the capital of the gambler after round $n$ on language $A$. The player wins on a language $A$ if he manages to make his capital arbitrarily large during the game, i.e. $\limsup _{n \rightarrow \infty} d\left(\chi_{A}[0 \ldots n]\right)=\infty$ and we say that martingale $d$ succeeds on $A$. The success set $S^{\infty}[d]$ of a martingale $d$ is the class of all languages on which $d$ succeeds.

Lutz's idea to define a measure notion on the class $E$ is to consider only martingales computable in a certain time bound, i.e. martingales $d$ such that $d(w)$ can be computed in time $2^{c|s| w \mid}$ for some $c>0$. Such a martingale is called 
E-computable ${ }^{1}$. E-computable martingales are the main tool for defining a measure notion on $\mathrm{E}$, as the following definition shows.

Definition 1 ([16]) A class $C$ has E-measure zero (also denoted p-measure zero) if there is an $\mathrm{E}$-computable martingale $d$ that succeeds on every language of $C$.

This property is monotone in the following sense: If class $D$ is contained in class $C$, and $C$ has E-measure zero, then $D$ has E-measure zero.

Definition 2 ([16]) A class $C$ has $\mathrm{E}$-measure one if its complement $\mathrm{E}-C$ has $\mathrm{E}$-measure zero.

It was shown in [16] that $\mathrm{E}$ does not have E-measure zero, which is known as the measure conservation property. Since finite unions of measure zero sets have measure zero it's impossible for a class to have both measure zero and one.

It was proved in [16] that enumerable infinite unions of measure zero sets have measure zero, more precisely.

Theorem 3 (Lutz) Suppose $\left\{d_{i}\right\}_{i \geq 1}$ is a set of martingales, each succeeding on class $C_{i}$; where $d(i, w):=d_{i}(w)$ is computable in time $2^{c|s| w \mid}+i^{c}$ for a some constant $c>0$. Then $\cup_{i \geq 1} C_{i}$ has E-measure zero.

The following result shows that approximable martingales can be replaced by exactly computable ones.

Lemma 4 Exact Computation Lemma [16]

Let $d:\{0,1\}^{*} \rightarrow \mathbb{R}_{+}$be a martingale such that there exists a family of approximations $\left\{\hat{d}_{k}\right\}_{k}$ where $\hat{d}_{k}(w)$ is computable in time $2^{c|s| w \mid}+k^{c}$ for some $c>0$, and such that $\left|\hat{d}_{k}(w)-d(w)\right| \leq 2^{-k}$. Then there exists an $\mathrm{E}$-computable martingale $d^{\prime}:\{0,1\}^{*} \rightarrow \mathbb{Q}_{+}$such that $S^{\infty}[d]=S^{\infty}\left[d^{\prime}\right]$.

For a survey on resource-bounded measure see [17].

\subsection{Resource-bounded Dimension}

Lutz's idea for defining a dimension notion via martingales, is to levy taxes on the martingales' wins, so that only martingales whose capital grows quickly are considered. This motivates the following definition.

$\overline{1}$ Equivalently, when the time bound is computed with respect to the input size $|w|$, the corresponding martingales are called $p$-computable. 
Definition 5 For a real number $s \geq 0$, a martingale is said to be $s$-successful on a language $A$, if $\lim \sup _{m \rightarrow \infty} \frac{d\left(\overline{A\left\lceil s_{m-1}\right)}\right.}{2^{(1-s) m}}=\infty$. A martingale is s-successful on a class if it is s-successful on every language of the class.

Remark 6 Similarly d is said strongly s-successful on A, if limsup in Definition 5 is replaced by lim inf.

The dimension of a class is defined as the largest tax rate which can be levied on the martingales' benefits, without preventing them from winning.

Definition 7 Let $C$ be any complexity class. The E-dimension of $C$ (resp. E-strong-dimension ) is the infimum over all $s \in[0,1]$, such that there exists an $\mathrm{E}$-computable martingale which s-succeeds (resp. strongly s-succeeds) on $C$.

It was shown in [18] that the E-dimension notion satisfies all three basic measure properties, namely that $E$ has $E$-dimension one, every language in $E$ has E-dimension zero, and finally enumerable infinite unions of sets of E-dimension $s$ have E-dimension $s$. More precisely,

Definition 8 Let $X, X_{0}, X_{1}, X_{2}, \cdots$ be complexity classes. $X$ is a $\mathrm{E}$-union of the $\mathrm{E}$ dimensioned sets $X_{0}, X_{1}, X_{2}, \cdots$ if $X=\bigcup_{k \geq 0} X_{k}$, and for each $s>$ $\sup _{k \in \mathbb{N}} \operatorname{dim}_{\mathbb{E}}\left(X_{k}\right)$, there is a function $d: \mathbb{N} \times\{0,1\}^{*} \rightarrow[0, \infty)$ with the following properties: $d$ is $\mathbf{E}$-computable, for each $k \in \mathbb{N}$, the function $d_{k}(w):=d(k, w)$ is a martingale, and for each $k \in \mathbb{N}, d_{k}$ s-succeeds on $X_{k}$.

The following Lemma states that the E-dimension of a E-union of sets is the supremum of the E-dimension of all sets.

Lemma 9 [18]

Let $X, X_{0}, X_{1}, X_{2}, \cdots$, be a E-union of the E-dimensioned sets $X_{0}, X_{1}, X_{2}, \cdots$. Then $\operatorname{dim}_{\mathrm{E}}(X)=\sup _{k \in \mathbb{N}} \operatorname{dim}_{\mathrm{E}}\left(X_{k}\right)$.

\section{Generic Density}

Genericity is defined via strategies, here is a definition.

Definition 10 A function $h:\{0,1\}^{*} \rightarrow\{0,1\}^{*} \cup\{\perp\}$ is a partial one-bit extension strategy, if for every string $\tau \in\{0,1\}^{*}$ either $h(\tau)$ is not defined, denoted $h(\tau)=\perp$, or $h$ extends $\tau$ by one bit i.e. $h(\tau)=\tau b$ with $b \in\{0,1\}$ (the bit $b$ is denoted $\operatorname{exth}(\tau))$.

For simplicity we use the word strategy for partial one-bit extension strategy. We denote $h(\tau) \downarrow$ whenever $h(\tau)$ is defined, i.e. $h(\tau) \neq \perp$. We say language $A$ 
meets strategy $h$ if $h(\tau) \sqsubset \chi_{A}$ for some string $\tau \in\{0,1\}^{*}$.

Several notions of genericity have been studied, based on different notions of extension strategies. Lutz and Fenner studied total strategies that extend the input by more than one bit $[15,8]$, whereas the strategies (partial one-bit extension) we consider were introduced by Ambos-Spies [1]. We are interested in a genericity notion on the class $\mathrm{E}$. This motivates the following definition.

Definition 11 Let $c>0$. A strategy $h:\{0,1\}^{*} \rightarrow\{0,1\}^{*} \cup\{\perp\}$ is $2^{c n}$ computable if there is a Turing machine which on input $\sigma$ computes $h(\sigma)$, in time $2^{c\left|s_{\sigma}\right|}$.

A strategy is E-computable if it is $2^{c n}$-computable for some $c>0$.

As mentioned earlier, we want to quantify the genericity notion of AmbosSpies [1]. This motivates the following definition.

Definition 12 Strategy $h$ is s-dense along some language $A$, with $s \in[0,1]$, if

$$
\limsup _{n \rightarrow \infty}\left|\left\{x \in\left\{s_{0}, s_{1}, \cdots, s_{n}\right\}: h(A 1 x) \downarrow\right\}\right|-s n=\infty .
$$

Remember that strategies are supposed to predict characteristic sequences of languages, so the higher the density of a strategy is, the more prediction it tries to make. $s$-strongly-dense is defined similarly with limsup replaced by liminf.

Let us introduce our notion of generic density.

Definition 13 A language $G$ is said $\left(s, 2^{c n}\right)$-generic if it meets every $2^{c n}$ computable strategy which is $(1-s)$-dense along $G$.

Informally $s$-generic sets cannot be predicted correctly by strategies, and the bigger $s$ is, the bigger the set of defeated strategy is. For $s$ close to 1 all strategies halting on at least a small portion of the strings are to be met, $s$ close to 0 is the other extreme, where only strategies halting on a huge fraction of all strings are to be met. For the genericity notion of Ambos-Spies [1], all strategies halting on at least infinitely many strings are to be met. $s$-stronglygeneric is defined similarly with $s$-dense replaced by $s$-strongly-dense.

Definition 14 Let $c>0$. The $2^{c n}$-generic density of a language $A$, denoted genfreq $_{2^{c n}}(A)$, is the supremum over all $s \in[0,1]$ such that $A$ is $\left(s, 2^{c n}\right)$ generic.

Intuitively the bigger the generic density of a sequence is, the more unpredictability it contains. 
Similarly the E-generic density of $A$, denoted $\operatorname{genfreq}_{\mathrm{E}}(A)$, is the sup over all $s \in[0,1]$ for which $A$ is $\left(s, 2^{c n}\right)$-generic for some $c>0$. Strong generic density genFreq $_{2^{c n}}(A)$ and genFreq $\mathrm{E}_{\mathrm{E}}(A)$ are defined by replacing generic with strongly generic in Definition 14.

The following result shows that $s$-generic sequences do exist for any computable $s$, but contrary to random sequences, they can be sparse.

Theorem 15 For every E-computable real $s \in[0,1]$ and every $c \geq 1$, there exists a sparse set $G \in \mathrm{E}$ such that $\operatorname{genfreq}_{2^{c n}}(G)=s$.

PROOF. Let $c \geq 1$ and $s \in[0,1]$ be an E-computable real, and denote by $\alpha_{n}$ the E-approximations of $s$, i.e.

$$
\left|s-\alpha_{n}\right| \leq \frac{1}{n}
$$

where $\alpha_{n}$ is computable in time polynomial in $n$. Let $\left\{h_{i}\right\}_{i}$ be an enumeration of all strategies computable in time $2^{c n}$, obtained by enumerating all corresponding alarm clocked Turing machines. Consider the following language $G$, whose characteristic sequence for strings of size $n$ is divided into two consecutive zones $R_{n}$ followed by $Q_{n}$; where $R_{n}$ contains $\left\lfloor\alpha_{n} 2^{n}\right\rfloor$ bits. $Q_{n}$ only contains 0 's, it's the easy zone of the language. $R_{n}$ is defined to prevent the first $n$ strategies from making correct predictions on its bits. Thus let $x$ be a string of size $n$ whose membership bit is in zone $R_{n}$, and suppose $G$ is already defined on previous strings. Find the least index $j \leq n$ such that $h_{j}$ has not been met on some previous string $y<x$, and such that $h_{j}(G 1 x) \downarrow$. If such a $j$ exists let

$$
G(x)=\operatorname{exth}_{j}(G 1 x)
$$

and call $h_{j}$ met on string $x$, otherwise let $G(x)=0$.

Computing $G(x)$ requires at most $2^{n+1}$ recursive steps, each requiring at most $n$ computation of $h_{j}$ on strings of size at most $n$; since $h_{j}$ is computable in time $2^{c n}, G \in \operatorname{DTIME}\left(2^{(c+2) n}\right)$.

Let us show that

$$
\operatorname{genfreq}_{2^{c n}}(G)=s .
$$

Let $\epsilon>0$. We start by showing that $G$ is not $\left(s+\epsilon, 2^{c n}\right)$-generic. Consider the following $2^{c n}$-computable strategy $h$ where $\operatorname{exth}\left(\begin{array}{lll}L & x\end{array}\right)=1$ whenever $x$ is a string whose membership bit is in $Q_{n}$, and $h$ is not defined on all other 
strings. $h$ is $(1-s-\epsilon)$-dense along $G$ since,

$$
\begin{aligned}
& \limsup _{n \rightarrow \infty}\left|\left\{x \in\left\{s_{0}, s_{1}, \cdots, s_{2^{n}-1}^{n}\right\}: h(G 1 x) \downarrow\right\}\right|-(1-(s+\epsilon))\left|\left\{s_{0}, s_{1}, \cdots, s_{2^{n}-1}^{n}\right\}\right| \\
& \geq \limsup _{n \rightarrow \infty} \sum_{j=0}^{n}\left(1-\alpha_{j}\right) 2^{j}-(1-(s+\epsilon)) \sum_{j=0}^{n} 2^{j} \\
& =\limsup _{n \rightarrow \infty} \sum_{j=0}^{n}\left(s-\alpha_{j}+\epsilon\right) 2^{j}=\infty
\end{aligned}
$$

Since $h$ is not met by $G$, this proves the first part. Let us show that $G$ is $\left(s-\epsilon, 2^{c n}\right)$-generic. Let $h$ be any $2^{c n}$-computable strategy and suppose for a contradiction that $h$ is $(1-s+\epsilon)$-dense along $G$ and $G$ does not meet $h$. It is clear that there can be only a constant number of $x$ such that $h(G 1 x) \downarrow$; otherwise suppose there are infinitely many such $x$. Then after a finite number of steps of $G$ 's construction, there will be a string $y$ such that $h_{j}=h$ (with $j \leq|y|$ ), all $h_{k}$ with $k<j$ will either have been met or will not halt on $G 1 y$, and $h_{j}(G 1 y) \downarrow$, thus

$$
G(y)=\operatorname{exth}(G 1 y)
$$

which is a contradiction. So suppose there are $K$ strings $x$ within all $R_{n}$ zones such that $h(G 1 x) \downarrow$, we have

$$
\begin{aligned}
& \limsup _{n \rightarrow \infty}\left|\left\{x \in\left\{s_{0}, s_{1}, \cdots, s_{2^{n}-1}^{n}\right\}: h(G 1 x) \downarrow\right\}\right|-(1-(s-\epsilon))\left|\left\{s_{0}, s_{1}, \cdots, s_{2^{n}-1}^{n}\right\}\right| \\
& \leq K+\limsup _{n \rightarrow \infty} \sum_{j=0}^{n}\left[\left(1-\alpha_{j}\right) 2^{j}+1\right]-(1-(s-\epsilon)) \sum_{j=0}^{n} 2^{j} \\
& \leq K+\limsup _{n \rightarrow \infty} \sum_{j=0}^{n}\left[\left(s-\alpha_{j}-\epsilon\right) 2^{j}+1\right]=-\infty
\end{aligned}
$$

i.e. $h$ is not $(1-s+\epsilon)$-dense along $G$, which is a contradiction. Thus $\operatorname{genfreq}_{2^{c n}}(G)=$ $s$.

Since $R_{n}$ contains at most $n$ bits equal to $1, G$ is sparse.

Theorem 16 For every EXP-computable real $s \in[0,1]$, there exists a sparse set $G \in \mathrm{EXP}$, such that $\operatorname{genfreq}_{\mathrm{E}}(G)=s$.

PROOF. The proof is similar to Theorem 15, except that $\left\{h_{i}\right\}_{i}$ is replaced by an enumeration of all strategies computable in time $2^{i n}$.

Remark 17 Similar arguments show that Theorem 15 also holds by replacing genfreq with genFreq. 


\section{Generic Density vs Resource-bounded Dimension}

As mentioned earlier, the strong generic density of a sequence is related to its strong dimension [5], more precisely every $s$-strongly random set is also $s$ strongly-generic, i.e. every set with a certain amount of randomness relatively to strong dimension, also contains a certain amount of unpredictability in regard to strong generic density.

Whereas $s$-generic sets are the typical sets for generic density, the following standard notion characterizes the typical sets for strong dimension.

Definition 18 Let $s \in[0,1]$. A language $R$ is $\left(s, 2^{c n}\right)$ strongly random if no martingale computable in $2^{\text {cn }}$ steps is strongly s-successful on $R$.

A set $R$ is $(s, \mathrm{E})$-strongly random if it is $\left(s, 2^{c n}\right)$-strongly random for every $c>0$.

$s$-strongly random sets are typical because they determine the E-strong dimension of a class that contains them, as the following standard result shows.

Lemma 19 Let $s \in[0,1], c>0$ and let $C$ be a class of languages such that $C$ does not contain any $\left(s, 2^{c n}\right)$-strongly random languages. Then $\operatorname{Dim}_{\mathrm{E}}(C) \leq s$.

PROOF. Let $s, c, C$ be as above, and let $\left\{d_{i}\right\}_{i}$ be an enumeration of all martingales computable in time $2^{c n}$, obtained by enumerating all alarm clocked Turing machines $\left\{M_{i}\right\}_{i}$ running in time $2^{c n}+i^{b}$ (where $b$ is some constant), and for $w \in\{0,1\}^{*}, b \in\{0,1\}$ letting

$$
d_{i}(w b)= \begin{cases}M_{i}(w b) & \text { if } M_{i}(w 0)+M_{i}(w 1)=2 M_{i}(w) \\ M_{i}(w) & \text { otherwise }\end{cases}
$$

Thus $d_{i}(w)$ is computable in time $2^{(c+2)\left|s_{|w|}\right|}+i^{b}$. Let

$$
d(w)=\sum_{i \geq 1} 2^{-i} d_{i}(w)
$$

The sum is convergent because $d_{i}(\lambda)=1$ and $d_{i}(w) \leq 2^{|w|}$ for every $i$. $d$ is a martingale because all the $d_{i}$ 's are. Since

$$
d(w) \geq 2^{-i} d_{i}(w)
$$


we have: $d_{i} s$-strongly succeeds on $L$ implies $d s$-strongly succeeds on $L$. Let

$$
\hat{d}_{k}(w)=\sum_{j=1}^{k+|w|} 2^{-j} d_{j}(w)
$$

$\hat{d}_{k}(w)$ is computable in time $2^{(c+4)\left|s_{|w|}\right|}+k^{b+1}$, and $\hat{d}_{k}$ is a $2^{-k}$-approximation of $d$ because

$$
\left|d(w)-\hat{d}_{k}(w)\right| \leq \sum_{j=k+|w|+1}^{\infty} 2^{-j} d_{j}(w) \leq 2^{|w|} \sum_{j=k+|w|+1}^{\infty} 2^{-j} \leq 2^{-k} .
$$

By the exact computation Lemma 4 there exists a martingale $d^{\prime}$ computable in time $2^{d n}$ for some $d>0$, such that

$$
d^{\prime}(w) \geq \frac{1}{2} d(w)
$$

thus $d_{i} s$-strongly succeeds on $L$ implies $d^{\prime} s$-strongly succeeds on $L$, for every $i \geq 1$. Suppose $d^{\prime}$ does not $s$-strongly succeed on $C$, i.e. there exists $L \in C$ such that $d^{\prime}$ does not $s$-strongly succeed on $L$. Thus for any $i \geq 1, d_{i}$ does not $s$-strongly succeed on $L$, i.e. $L$ is $\left(s, 2^{c n}\right)$-strongly random which contradicts the assumption on $C$.

Corollary 20 Lemma 19 still holds if we replace strongly random with random and Dim with dim.

The following result shows that every $s$-strongly random set is $s$-stronglygeneric, i.e. quantified randomness implies quantified unpredictability. We prove a more general result in Section 6 .

Theorem 21 Let $c>0$. Let $R$ be $\left(s, 2^{(c+2) n}\right)$-strongly random, then $R$ is $\left(s, 2^{\text {cn }}\right)$-strongly-generic.

PROOF. Follows from Corollary 27.

Corollary 22 Let $c>0$. Let $R$ be $\left(s, 2^{(c+2) n}\right)$-random, then $R$ is $\left(s, 2^{c n}\right)$ generic.

PROOF. Similar to Theorem 21.

The converse of Theorem 21 is not true as the following section shows. 


\section{Comparing the Density Notions}

As the following result shows, quantified unpredictability and quantified randomness are different notions.

Theorem 23 There exists a language $S$ such that $\operatorname{Dim}_{\mathrm{E}}(S)<\operatorname{genfreq}_{\mathrm{E}}(S)$ and

$$
\begin{gathered}
\operatorname{Dim}_{\mathrm{E}}(S)<\operatorname{genFreq}_{\mathrm{E}}(S) \\
\vee \\
\vee \\
\operatorname{dim}_{\mathrm{E}}(S)<\operatorname{genfreq}_{\mathrm{E}}(S)
\end{gathered}
$$

And for any sequence $S$, Equation 2 holds with less or equal inequalities.

PROOF. Equation 2 with less or equal inequalities holds by definition. For strict inequalities, consider the following language $S$ whose characteristic sequence for strings of size $n$ starts with $\frac{3}{4} 2^{n}$ bits 1 followed by a zone of length $\frac{1}{8} 2^{n}$ containing at most $n$ generic bits as in Theorem 15 (obtained by trying to meet all among the $n$ first E-computable strategies which are dense along $S$ outside the " $\frac{3}{4}$ zone") and all other bits equal to 1 . The last zone of length $\frac{1}{8} 2^{n}$ contains only random bits i.e. such that the capital of martingale $d$ stays bounded, where

$$
d(w)=\sum_{j \geq 1} 2^{-j} d_{j}(w)
$$

is the sum of all E-computable martingales, thus preventing any E-computable martingale from winning money on those bits; more precisely, let $x$ be a string whose membership bit is in the last zone, and let

$$
S(x)=1 \text { iff } d((S \uparrow x) 1) \leq d((S \uparrow x) 0) .
$$

Let $\epsilon>0$. It is easy to see that the strategy $h$ which outputs 0 only in the $\frac{3}{4}$ zone is $\left(\frac{3}{4}-\epsilon\right)$-strongly-dense along any language and is not met by $S . h$ is $\left(\frac{6}{7}-\epsilon\right)$-dense because after the $\frac{3}{4}$ zone of strings of size $n, h$ halted on $\frac{3}{4}$ of all strings of size less than $n$ plus on $\frac{3}{4} 2^{n}$ strings of size $n$, among a total of $2^{n}+\frac{3}{4} 2^{n}$ strings. Thus $h$ is $\left(\frac{6}{7}-\epsilon\right)$-dense. The generic and random bits outside the $\frac{3}{4}$ zone ensure that every E-computable strategy halting infinitely often in this zone would be eventually met, thus

$$
\operatorname{genFreq}_{\mathrm{E}}(S)=\frac{1}{4}
$$

and

$$
\operatorname{genfreq}_{\mathrm{E}}(S)=\frac{1}{7}
$$


Consider martingale $d$ that bets a fraction $a$ (with $0<a<1$ ) of its current capital that the membership bit of the first $\frac{7}{8}$ strings of size $n$ is 1 . Whenever $d$ 's bet is correct i.e. outside the $n$ generic bits, $d$ 's capital is multiplied by a factor $1+a$, respectively by a factor $1-a$ on the $n$ generic bits. Thus for the strong dimension, after having bet on all strings of size at most $n$, $d$ 's capital is greater than

$$
(1+a)^{\frac{7}{8} 2^{n+1}-n^{2}}(1-a)^{n^{2}}
$$

thus $d\left(\frac{1}{8}+\epsilon\right)$-succeeds on $S$ in the liminf by an appropriate choice of $a$. For the limsup, after having bet on the $\frac{7}{8} 2^{n}$ first strings of size $n$, d's capital is greater than

$$
(1+a)^{\frac{7}{8} 2^{n+1}-n^{2}}(1-a)^{n^{2}}
$$

thus $d\left(\frac{1}{15}+\epsilon\right)$-succeeds on $S$ in the limsup by an appropriate choice of $a$. The random bits in the last zone ensure that no E-computable martingale can win more than a bounded amount of money on those bits, thus

$$
\operatorname{Dim}_{\mathrm{E}}(S)=\frac{1}{8} \text { and } \operatorname{dim}_{\mathrm{E}}(S)=\frac{1}{15}
$$

As the previous result shows, there exists a set whose strong dimension is smaller than its generic density. The following result shows that the converse also holds, i.e. these two notions are incomparable.

Theorem 24 There exists a languages $S$ such that

$$
\operatorname{Dim}_{\mathrm{E}}(S)=1 \text { and } \operatorname{genfreq}_{\mathrm{E}}(S)=0 .
$$

PROOF. Consider the following language $S$ whose characteristic sequence is a succession of blocs $B_{0} B_{1} B_{2} \cdots$ where block $B_{i}$ has size $f(i)$, defined as follows. First $f(0)=1$. Zone $B_{2 i}$ contains only random bits, such that the capital of $d$ stays bounded on those bits, where

$$
d(w)=\sum_{j \geq 1} 2^{-j} d_{j}(w)
$$

is the sum of all E-computable martingales, thus preventing any E-computable martingale from winning money on those bits. For the size of $B_{2 i}$, let $f(2 i)$ be large enough such that the capital of any E-computable martingale betting on strings up to $B_{2 i}$ with tax rate $1 / i$ gets smaller than 1 ; since such a martingale can at most double its capital after each string, let $f(2 i)$ be the smallest integer $I$ such that

$$
\frac{2^{g(2 i-1)}}{2^{\frac{1}{i}(g(2 i-1)+I)}} \leq 1
$$


where

$$
g(i)=\sum_{j=0}^{i} f(j)
$$

Block $B_{2 i+1}$ contains only 1's, and has size $f(2 i+1)$, which is defined as the smallest integer ensuring that the strategy $h$ that outputs 0 in every $B_{2 i+1}$ is $\left(1-\frac{1}{i}\right)$-dense along $S$; i.e. the smallest integer $I$ such that

$$
\sum_{j=0}^{i-1} f(2 j+1)+I-\left(1-\frac{1}{i}\right)(g(2 i)+I)>i .
$$

By construction of $S$ no E-computable martingale can $\left(1-\frac{1}{i}\right)$-win on $S$ in the liminf (for every $i$ ), thus $\operatorname{Dim}_{\mathrm{E}}(S)=1$. On the other hand, the E- computable strategy $h$ is $\left(1-\frac{1}{i}\right)$-dense along $S$ for every $i$, and is not met by $S$; thus $\operatorname{genfreq}_{\mathrm{E}}(S)=0$.

Theorem 23 and 24 yield the following corollary.

Corollary 25 Dim $_{\mathrm{E}}$ and genfreq $\mathrm{E}_{\mathrm{E}}$ are incomparable.

\section{Generic Density under Different Probability Measures}

In this section we highlight a main feature of generic density over resourcebounded dimension, that is whereas the latter notion depends on the underlying probability measure, generic density does not. As we shall see, this implies that dimension results obtained by generic density methods, are somehow more informative, because they hold in a wide variety of probability measure spaces. Let us give some preliminary definitions from [5]. A probability measure on the Cantor space is a function $\nu:\{0,1\}^{*} \rightarrow[0,1]$ such that $\nu(\lambda)=1$ and for all strings $w, \nu(w)=\nu(w 0)+\nu(w 1)$. Informally, $\nu(w)$ is the probability that $w \sqsubset L$, where the sequence $L$ is chosen according to $\nu$. A bias sequence is a sequence $\vec{\beta}=\left(\beta_{0}, \beta_{1}, \ldots\right)$ of real numbers $\beta_{i} \in[0,1]$. Intuitively, $\beta_{i}$ is the probability that the $i$ th toss of a biased coin yields 1 . For a bias sequence $\vec{\beta}$, define the $\vec{\beta}$-probability measure on $C$ by $\mu^{\vec{\beta}}(w)=\prod_{i=0}^{|w|-1} \beta_{i}(w)$, where $\beta_{i}(w)=\beta_{i}$ if $w_{i}=1$ and $1-\beta_{i}$ otherwise. $\mu^{\vec{\beta}}$ represents the probability that some language $L$ satisfies $w \sqsubset L$, where the $i$ th bit of $L$ is determined by a coin toss with bias $\beta_{i}$. For simplicity $\mu^{\vec{\beta}}$ is sometimes denoted $\vec{\beta}$. The usual probability measure is called the uniform probability measure, denoted $\mu(w)=2^{-|w|}$, and corresponds to the toss of a fair coin. 
Resource-bounded dimension on spaces with probability measure $\nu$ is defined via $\nu$-s-gales, here is a definition.

Definition 26 [5] Let $\nu$ be a probability measure on $\mathrm{C}$, let $s \in[0,1]$ and $t(n) \geq$ $2^{O(n)}$ be a time bound. A $t(n)$-computable $\nu$-s-gale is a function $d:\{0,1\}^{*} \rightarrow$ $[0, \infty)$ such that for all strings $w, d(w) \nu^{s}(w)=d(w 0) \nu^{s}(w 0)+d(w 1) \nu^{s}(w 1)$ and $d(w)$ is computable in $t\left(\left|s_{|w|}\right|\right)$ steps.

Intuitively the $s$ in Definition 26 represents the tax levied on the martingale's wins, whereas the factors $\nu$ adjust the wins according to the probability measure $\nu$ : if some bit appears with higher probability, then the payoff while betting on this bit ought to be smaller. An E-computable $\nu$-s-gale is a $t(n)$ computable $\nu$-s-gale for some $t(n)=2^{O(n)}$.

Similarly to the usual notion, the $\mathrm{E}$ - $\nu$-dimension of a language $L$, denoted $\operatorname{dim}_{\mathrm{E}}^{\nu}(L)$, is the infimum over all $s$ such that there is an E-computable $\nu$-s-gale $d$ such that $\lim \sup _{m \rightarrow \infty} d(L 1 \mathrm{~m})=\infty$. It is easy to check that Lemma 19 also holds in spaces with any biased coin based probability measure.

For $s \in[0,1]$, denote by $\operatorname{DIM}_{\mathrm{E}}^{\nu}(\geq s)\left(\right.$ resp. $\left.\operatorname{GENfreq}_{\mathrm{E}}(\geq s)\right)$ the set of languages with E- $\nu$-dimension (resp. E-generic density) at least $s$. Denote by PDIM the strong dimension analogue and GENFreq the strong-genericity analogue.

The following result requires the weighted binary entropy function $H:(0,1)^{2} \rightarrow$ $[0, \infty)$ where $H(x, y)=x \log \frac{1}{y}+(1-x) \log \frac{1}{1-y}$ which is continuous on $(0,1)^{2}$.

It is clear by the work of $[19,5,9]$ that resource-bounded dimension depends on the underlying probability measure, i.e. for two bias sequences $\vec{\alpha}, \vec{\beta}$ converging to different values, $\operatorname{DIM}_{\mathrm{E}}^{\vec{\alpha}}(\geq s) \neq \operatorname{DIM}_{\mathrm{E}}^{\vec{\beta}}(\geq s)$, i.e. sequences with high dimension in a space with underlying probability $\vec{\alpha}$ can have smaller dimension in a space with underlying probability $\vec{\beta}$.

The following result shows that this is not the case for generic density. More precisely we show that for a sequence of biases $\vec{\beta}$ converging to some number $\beta$, the sequences with $\vec{\beta}$-dimension $s \log (1 / \beta) / H(s, \beta)$ have generic density $s$. The factor $\log (1 / \beta) / H(s, \beta)$ that appears when going from dimension to generic density is because the payoffs are not equal whether the bit that is bet on is zero or one, i.e. if for example $\beta<1 / 2$ then the probability of the bit 0 is bigger, therefore the payoff on such bits is smaller. So if the bits of the non-generic i.e. easily predicted sequence, are always predicted to be 0 , the dimension has to drop, which explains this factor. Note that when $\beta=1 / 2$, the factor is equal to 1 , i.e. disappears.

The following result highlights an advantage of the generic density method over the martingales based one, for dimension results that are proved by showing that a class contains an $s$-generic set. Such results simultaneously hold in a 
large range of biased-coin based probability measure spaces. Such an example is given in Section 7. Note that of course such an approach is not always possible for dimension results, see for example [5].

Theorem 27 Let $\vec{\beta}=\left(\beta_{0}, \beta_{1}, \ldots\right)$ be an $\mathrm{E}$-computable bias sequence, converging to $\beta \in\left(0, \frac{1}{2}\right]$. Let $s \in[0,1]$, then $\operatorname{GENfreq}_{\mathrm{E}}(\geq s) \supseteq \operatorname{DIM}_{\mathrm{E}}^{\vec{\beta}}\left(\geq s \frac{\log (1 / \beta)}{H(s, \beta)}\right)$.

PROOF. Let $\vec{\beta}, \beta, s$ be as above. Let $\epsilon>0$. Let $L \in \operatorname{DIM}_{\mathrm{E}}^{\vec{\beta}}\left(\geq \frac{s \log (1 / \beta)}{H(s, \beta)}\right)$. Let $F:[0,1] \times\left(0, \frac{1}{2}\right] \rightarrow[0, \infty)$, where $F(x, y)=\frac{x \log \frac{1}{y}}{H(x, y)} . F$ is continuous on $[0,1] \times\left(0, \frac{1}{2}\right],\left(H(x, y) \neq 0\right.$ on $\left.[0,1] \times\left(0, \frac{1}{2}\right]\right)$. Let $t=F(s, \beta)+\epsilon$, wlog $t$ is E-computable. By continuity of $F$, let $\delta>0$ such that $t>F\left(s^{\prime}, \beta^{\prime}\right)$ whenever $\left|s-s^{\prime}\right| \leq 2 \delta$ and $\left|\beta-\beta^{\prime}\right| \leq 2 \delta$. Suppose $L \notin \operatorname{GENfreq}_{\mathrm{E}}(\geq s)$, i.e. $L$ is not $(s+\delta)$-generic, and let $h$ be an E-strategy, $(1-s-\delta)$-dense along $L$, not met by $L$.

Let us construct an E-computable $\vec{\beta}$-t-gale that succeeds on $L$ contradicting the assumption on $L$. Denote $\nu=\mu^{\vec{\beta}}$ the probability measure induced by $\vec{\beta}$. For $w \in\{0,1\}^{*}$ and $b \in\{0,1\}$, let $\nu(w b \mid w)=\frac{\nu(w b)}{\nu(w)}$ and

$$
d(w b)= \begin{cases}d(w) \nu(w b \mid w)^{-t} & \text { if } \operatorname{exth}(w)=\bar{b} \\ 0 & \text { if } \operatorname{exth}(w)=b \\ d(w) \nu(w b \mid w)^{1-t} & \text { if } h(w)=\perp\end{cases}
$$

It is easy to check that $d$ is an E-computable $\vec{\beta}$-t-gale. Since the sequence $\vec{\beta}$ converges to $\beta$, let $I$ be such that $\left|\beta_{i}-\beta\right|<\delta$, whenever $i>I$. Let us compute a lower bound for $d$ 's wins. Suppose whenever $h(w)$ is defined, $b=1-\operatorname{exth}(w)$ is the bit for which payoffs are smallest, i.e. the capital of $d$ is increased by a factor $\left(1-\beta_{|w|+1}\right)^{-t}$ (if $\left.\beta_{|w|+1} \leq 1 / 2\right)\left(\operatorname{resp} .\left(\beta_{|w|+1}\right)^{-t}\left(\right.\right.$ if $\left.\beta_{|w|+1} \geq 1 / 2\right)$ ); and decreased by a factor $\left(\beta_{|w|+1}\right)^{1-t}$ (if $\left.\beta_{|w|+1} \leq 1 / 2\right)$ (resp. $\left(1-\beta_{|w|+1}\right)^{1-t}$ (if $\left.\beta_{|w|+1} \geq 1 / 2\right)$ ). In both cases, the increase factor is greater than $(1-(\beta-\delta))^{-t}$, and the decrease factor is greater than $(\beta-\delta)^{1-t}$ (because the resp. case can only happen when $\beta=1 / 2)$. Since $h$ is $(1-s-\delta)$-dense along $L$

$$
\exists^{\infty} n:\left|\left\{0 \leq j \leq n: \operatorname{exth}\left(L 1 s_{j}\right)=1\right\}\right|>(1-(s+\delta))(n+1) .
$$

Let $n \in \mathbb{N}$ be such that Equation 4 holds, and $s_{n}>I$. Whenever $y>I$, we 
have $\left|\beta_{|(L \mid y)|}-\beta\right|<\delta$, thus for $x=s_{n}$ we have

$$
\begin{aligned}
d(L \uparrow x) & \geq c \prod_{y \leq x, y>I, \operatorname{exth}(L \mid y)=\perp}(\beta-\delta)^{1-t} \prod_{y \leq x, y>I, \operatorname{exth}(L \mid y) \neq \perp}(1-(\beta-\delta))^{-t} \\
& \geq c\left[(\beta-\delta)^{1-t}\right]^{(s+\delta) \mid(L\lceil x) \mid}\left[(1-(\beta-\delta))^{-t}\right]^{(1-(s+\delta)) \mid(L\lceil x) \mid-I} \\
& \geq c^{\prime}\left[(\beta-\delta)^{s+\delta}\left((\beta-\delta)^{-(s+\delta)}(1-(\beta-\delta))^{s+\delta-1}\right)^{t}\right]^{(L\lceil x) \mid} \\
& =c^{\prime}\left[(\beta-\delta)^{s+\delta} 2^{t H(s+\delta, \beta-\delta)}\right]^{\mid(L\lceil x) \mid}
\end{aligned}
$$

for some constants $c=c(I)>0, c^{\prime}=c^{\prime}(I)>0$. By definition of $t,(\beta-$ $\delta)^{s+\delta} 2^{t H(s+\delta, \beta-\delta)}>1$, thus $d$ grows unbounded on $L$.

Corollary 28 The same holds by replacing $\operatorname{GENfreq}_{\mathrm{E}}(\geq s)$ with $\mathrm{GENFreq}_{\mathrm{E}}(\geq$ s) and $\operatorname{DIM}_{\mathrm{E}}^{\vec{\beta}}\left(\geq s \frac{\log (1 / \beta)}{H(s, \beta)}\right)$ with $\operatorname{PDIM}_{\mathrm{E}}^{\vec{\beta}}\left(\geq s \frac{\log (1 / \beta)}{H(s, \beta)}\right)$.

\section{Small Span Theorem in Dimension}

In this section we prove a small span theorem for bounded-truth-table reduction, in the strong dimension setting, in spaces with any biased coin based probability measure. The proof is adapted from [4] combined with results of the previous sections. This is an example where the generic density method is more informative than the martingale based approach, because we simultaneously prove the result for any biased coin based probability measure.

To clarify the proofs, we assume that all bounded truth-table reductions are in the following normal form, where all queries are ordered in decreasing order, and redundant ones are replaced by $\lambda$.

Definition $29 A$ - $k$-tt reduction $f\left(g_{1}, \ldots, g_{k}\right)$ is normal if for every $x \in$ $\{0,1\}^{*}$ there exists $k^{\prime} \leq k$ such that $g_{i}(x)>g_{i+1}(x)$ for $1 \leq i \leq k^{\prime}$, and $g_{i}(x)=\lambda$ for all $i \geq k^{\prime}$.

It is easy to check that any $p$ - $k$-tt reduction $f\left(g_{1}, \ldots, g_{k}\right)$ can be transformed into an equivalent normal reduction.

Definition 30 The collision set of a $p$ - $k$-tt reduction $f\left(g_{1}, \ldots, g_{k}\right)$ denoted Coll $(f)$ is the set of strings $x$, for which there exists $y<x$ such that $g_{i}(x)=$ $g_{i}(y)($ for $i=1, \ldots, k)$ and $f_{x}=f_{y}$, where $f_{x}=f(x, \cdot, \ldots, \cdot)$.

A $p$ - $k$-tt reduction $f\left(g_{1}, \ldots, g_{k}\right)$ is consistent with some language $A$, if for all strings $x, y$ s.t. $g_{i}(x)=g_{i}(y)$ (for $i=1, \ldots, k$ ) and $f_{x}=f_{y}$, we have $A(x)=A(y)$.

Definition 31 Let $c>0$ be some constant, and let $f\left(g_{1}, \cdots, g_{k}\right)$ be a $p$-k-tt 
reduction. The c-rank of $f\left(g_{1}, \cdots, g_{k}\right)$ is the largest integer $1 \leq r \leq k$ such that $\exists^{\infty} x: \frac{|x|-c}{k} \leq\left|g_{r}(x)\right|$. The c-rank is zero if no such integer exists.

The following Lemma states that the composition of two tt-reducibilities, is a tt-reducibility.

Lemma 32 [Folklore] Let $k \geq 1$ and $A, B, C$ be languages such that $A \geq_{k-t t}^{p}$ $B \geq_{k-t t}^{p} C$. Then $A \geq_{k^{2}-t t}^{p} C$.

The following is the main result of this section.

Theorem 33 (Small span theorem) Let $\vec{\beta}=\left(\beta_{0}, \beta_{1}, \ldots\right)$ be an $\mathrm{E}$-computable bias sequence, converging to $\beta \in\left(0, \frac{1}{2}\right]$. Let $A$ in $\mathrm{E}$ be any language, and $k \in \mathbb{N}$. Then either $\operatorname{Dim}_{\mathrm{E}}^{\vec{\beta}}\left(A^{\geq_{k-t t}^{p}} \cap \mathrm{E}\right)=0$ or $\mu_{\mathrm{E}}^{\vec{\beta}}\left(A^{\leq_{k-t t}^{p}}\right)=0$.

PROOF. Let $\epsilon>0, k \geq 1$, and let $A \in \operatorname{DTIME}\left(2^{a n}\right)$, for some $a>0$. Suppose $A^{\geq_{k-t t}^{p}} \cap \mathrm{E}$ contains no $\left(\epsilon, 2^{2 n}\right)$-strongly-generic set, then it contains no $\left(\epsilon \frac{\log \frac{1}{\beta}}{H(\epsilon, \beta)}, 2^{a^{\prime} n}\right)$-strongly $\vec{\beta}$-random set by Corollary 27 , for some $a^{\prime}>0$. An extension of Lemma $19 \operatorname{implies} \operatorname{Dim}_{\mathrm{E}}^{\vec{\beta}}\left(A^{\geq_{k-t t}^{p}} \cap \mathrm{E}\right) \leq \epsilon \frac{\log \frac{1}{\beta}}{H(\epsilon, \beta)}$. Since $\epsilon$ is arbitrary we are done.

Otherwise suppose $A^{\geq_{k-t t}^{p}} \cap \mathrm{E}$ contains an $\left(\epsilon, 2^{2 n}\right)$-strongly-generic set $G$, where $G \in \operatorname{DTIME}\left(2^{d n}\right)$. Let us show that the upper span of $A$ does not contain any $\left(1,2^{2 d\left(k^{2} n+c\right)}\right)$-generic set. Let $B \in A^{\leq_{k-t t}^{p}}$ be any language. Let $f\left(g_{1}, \cdots, g_{k^{2}}\right)$ be a normal $p$ - $k^{2}$-tt reduction of $G$ to $B$ of minimal $c$-rank (such a reduction exists by Lemma 32), where $c=c(\epsilon, k)$ is some constant to be determined later. Let us show that $B$ is not $\left(1,2^{2 d\left(k^{2} n+a\right)}\right)$-generic. Suppose

$$
\forall^{\infty} n: \mid\left\{\operatorname{Coll}(f) \cap\left\{s_{0}, \ldots, s_{n-1}\right\} \mid \geq\left(1-\frac{\epsilon}{2}\right) n .\right.
$$

Consider the following strategy $h$, where for any language $L$, exth $(L 1 y)=$ $1-L(x)$ whenever $y \in \operatorname{Coll}(f)$, and $x<y$ is the first string witnessing this fact. $h$ is $(1-\epsilon)$-strongly-dense along any language by Equation 5 , and $h$ is computable in $2^{2 n}$ steps. Since

$$
\begin{aligned}
\operatorname{exth}(G \uparrow y) & =1-G(x)=1-f\left(x, B\left(g_{1}(x)\right), \ldots, B\left(g_{k^{2}}(x)\right)\right) \\
& =1-f\left(y, B\left(g_{1}(y)\right), \ldots, B\left(g_{k^{2}}(y)\right)\right)=1-G(y)
\end{aligned}
$$

whenever $h(G 1 y)$ is defined, $h$ is not met by $G$, which contradicts the $\left(\epsilon, 2^{2 n}\right)$ strong-genericity assumption on $G$. Thus the negation of Equation 5 holds, which can be reformulated as:

$$
\exists^{\infty} n: \mid\left\{\overline{\operatorname{Coll}}(f) \cap\left\{s_{0}, \ldots, s_{n-1}\right\} \mid \geq \frac{\epsilon}{2} n\right.
$$


Let us show that

$$
\exists^{\infty} x: \frac{|x|-c}{k^{2}} \leq\left|g_{1}(x)\right| .
$$

Let $F_{m}$ denote the set of tuples $\left(j, x_{1}, \ldots, x_{k^{2}}\right)$ where $j$ is a $k^{2}$-variable Boolean function, and $\left|x_{i}\right|<m$ for $1 \leq i \leq k^{2}$. Because there are $2^{2^{k^{2}}}$ such Boolean functions, and $2^{m}-1$ strings of size less than $m,\left|F_{m}\right|<2^{2^{k^{2}}} 2^{k^{2} m}$. If $x$ is a string such that $\left|g_{1}(x)\right|<m$ then $\left(f_{x}, g_{1}(x), \ldots, g_{k^{2}}(x)\right) \in F_{m}$ and if $x, y \in \overline{\operatorname{Coll}}(f)$ then

$$
\left(f_{x}, g_{1}(x), \ldots, g_{k^{2}}(x)\right) \neq\left(f_{y}, g_{1}(y), \ldots, g_{k^{2}}(y)\right) .
$$

Let $N$ be such that Equation 6 holds, and let $n=\lfloor\log N\rfloor$. Let $m=m(n)=$ $\left\lfloor\frac{n-b}{k^{2}}\right\rfloor$, where $b\left(\epsilon, k^{2}\right)$ is some constant ensuring that $\left|F_{m}\right|<\frac{\epsilon}{4} N$. Let $x \in$ $\overline{\operatorname{Coll}}(f) \cap\left\{s_{0}, \ldots, s_{N-1}\right\}$, be the largest string such that $\left(f_{x}, g_{1}(x), \ldots, g_{k^{2}}(x)\right) \notin$ $F_{m}$, such a string exists by Equation 6 moreover it has size greater than $\log \left(\frac{\epsilon}{4} N\right)$. Since $\left(f_{x}, g_{1}(x), \ldots, g_{k^{2}}(x)\right) \notin F_{m}$, we have $\left|g_{1}(x)\right|>m=\left\lfloor\frac{n-b}{k^{2}}\right\rfloor \geq$ $\frac{|x|-c}{k^{2}}$ for some constant $c=c(\epsilon, k)$. Since Equation 6 holds for infinitely many $N$, this proves Equation 7, i.e $f$ has $c$-rank $>0$.

Let us show that

$$
\begin{aligned}
\exists^{\infty} x: & \frac{|x|-c}{k^{2}} \leq\left|g_{1}(x)\right| \text { and, } \\
& f_{x}\left(0, B\left(g_{2}(x)\right), \ldots, B\left(g_{k^{2}}(x)\right)\right) \neq f_{x}\left(1, B\left(g_{2}(x)\right), \ldots, B\left(g_{k^{2}}(x)\right)\right) .
\end{aligned}
$$

Otherwise suppose Equation 8 fails, i.e.

$$
\begin{aligned}
\forall x>n_{0}: & \frac{|x|-c}{k^{2}}>\left|g_{1}(x)\right| \text { or, } \\
& f_{x}\left(0, B\left(g_{2}(x)\right), \ldots, B\left(g_{k^{2}}(x)\right)\right)=f_{x}\left(1, B\left(g_{2}(x)\right), \ldots, B\left(g_{k^{2}}(x)\right)\right) .
\end{aligned}
$$

Consider the following $p$ - $k^{2}$-tt reduction $f^{\prime}\left(g_{1}^{\prime}, \ldots, g_{k^{2}}^{\prime}\right)$. Let $x$ be a string such that $n_{0}<\frac{|x|-c}{k^{2}} \leq\left|g_{1}(x)\right|$. Define

$$
\left(g_{1}^{\prime}(x), \ldots, g_{k^{2}}^{\prime}(x)\right)=\left(g_{2}(x), \ldots, g_{k^{2}}(x), \lambda\right)
$$

and

$$
f_{x}^{\prime}\left(a_{1}, \cdots, a_{k^{2}}\right)=f_{x}\left(0, a_{1}, \ldots, a_{k^{2}-1}\right)
$$

for any such $x$, and let $\left(g_{1}^{\prime}(x), \ldots, g_{k^{2}}^{\prime}(x)\right)=\left(g_{1}(x), \ldots, g_{k^{2}}(x)\right)$ and $f_{x}^{\prime}=f_{x}$ for all other $x$. For the infinitely many strings $x$ satisfying $n_{0}<\frac{|x|-c}{k^{2}} \leq\left|g_{1}(x)\right|$, we have

$$
\begin{aligned}
f_{x}^{\prime}\left(B\left(g_{1}^{\prime}(x)\right), \ldots, B\left(g_{k^{2}}^{\prime}(x)\right)\right) & =f_{x}\left(0, B\left(g_{2}(x)\right), \ldots, B\left(g_{k^{2}}(x)\right)\right) \\
& =f_{x}\left(b, B\left(g_{2}(x)\right), \ldots, B\left(g_{k^{2}}(x)\right)\right)
\end{aligned}
$$

for every bit $b \in\{0,1\}$ hence

$$
f_{x}^{\prime}\left(B\left(g_{1}^{\prime}(x)\right), \ldots, B\left(g_{k^{2}}^{\prime}(x)\right)\right)=f_{x}\left(B\left(g_{1}(x)\right), B\left(g_{2}(x)\right), \ldots, B\left(g_{k^{2}}(x)\right)\right)=G(x)
$$


i.e. $G$ is $p$ - $k^{2}$-tt reducible to $B$ via $f^{\prime}$, where $f^{\prime}$ is normal, and for all infinitely many $x$ such that $n_{0}<\frac{|x|-c}{k^{2}} \leq\left|g_{1}(x)\right|, f^{\prime}$ is obtained from $f$ by dropping the largest query $g_{1}(x)$. Therefore the $c$-rank of $f^{\prime}$ is equal to $c$-rank $(f)-1$, which contradicts the minimality of the $c$-rank of $f$.

Let us show that Equation 8 implies that $B$ is not $\left.\left(1,2^{2 d\left(k^{2} n+a\right)}\right)\right)$-generic. Consider the following strategy $h$, where for any language $L, h\left(\begin{array}{lll}L & y\end{array}\right)$ is defined as follows. Let $x$ be the first string with $|x| \leq k^{2}|y|+a$, such that $g_{1}(x)=y$, and such that

$$
\underbrace{f_{x}\left(0, L\left(g_{2}(x)\right), \ldots, L\left(g_{k^{2}}(x)\right)\right)}_{=v_{0}} \neq \underbrace{f_{x}\left(1, L\left(g_{2}(x)\right), \ldots, L\left(g_{k^{2}}(x)\right)\right)}_{=v_{1}}
$$

whenever such a string $x$ exists. Since $g_{i}(x)<y$ (for $\left.i=2, \ldots, k^{2}\right), v_{0}$ and $v_{1}$ are computable in $2^{2|y|}$ steps. Let $i \in\{0,1\}$ such that $G(x)=v_{i}$, and let $\operatorname{ext} h(L 1 y)=1-i . h$ is computable in $2^{2 d\left(k^{2} n+a\right)}$ steps. By Equation $8, h$ is 0 -dense along $B$, and $h$ is not met by $B$, i.e. $B$ is not $\left.\left(1,2^{2 d\left(k^{2} n+a\right)}\right)\right)$-generic.

Thus $B$ is not $\left.\left(1,2^{2 d\left(k^{2} n+a\right)}\right)\right)-\vec{\beta}$-random by Theorem 27 . Hence $\mu_{\mathrm{E}}^{\vec{\beta}}\left(A^{\leq_{k-t t}^{p}}\right)=0$, as a corollary of the proof of Corollary 20 .

\section{Conclusion}

We have introduced a refined notion of genericity, in the same sense that resource-bounded dimension is a refinement of resource-bounded measure. We have exhibited a relationship between generic density and dimension, as well as a main difference regarding the underlying probability measure of the Cantor space, with the consequence that generic density based proofs are in some sense more informative than martingale based ones. We give an example of such a proof by showing a small span theorem in any (biased coin) probability measure space, for stronger reductions as previously considered in the dimension setting. We expect generic density to be useful for further resourcebounded dimension investigations.

Acknowledgments. I thank J. Lutz for suggesting the possible measure probability independence of generic density, and for bringing [13] to my attention, and the anonymous referees for their many helpful comments.

\section{References}

[1] K. Ambos-Spies. Resource-bounded genericity. Proceedings of the Tenth Annual Structure in Complexity Theory Conference, pages 162-181, 1995. 
[2] K. Ambos-Spies and E. Mayordomo. Resource-bounded measure and randomness. Lecture Notes in Pure and Applied Mathematics, pages 1-47, 1997.

[3] K. Ambos-Spies, W. Merkle, J. Reimann, and F. Stephan. Hausdorff dimension in exponential time. In Proceedings of the 16th IEEE Conference on Computational Complexity, 172:210-217, 2001.

[4] K. Ambos-Spies, H. Nies, and S. Terwijn. Genericity and resource bounded measure for exponential time. Theoretical Computer Science, 168:3-19, 1996.

[5] Krishna B. Athreya, John M. Hitchcock, Jack H. Lutz, and Elvira Mayordomo. Effective strong dimension in algorithmic information and computational complexity. Proceedings of the Twenty-First Symposium on Theoretical Aspects of Computer Science, pages 632-643, 2004.

[6] H. Buhrman and D. van Melkebeek. Hard sets are hard to find. Journal of Computer and System Sciences, 59(2):327-345, 1999.

[7] J. Dai, J.I. Lathrop, J.H. Lutz, and E. Mayordomo. Finite-state dimension. Theoretical Computer Science, 310:1-33, 2004.

[8] S. A. Fenner. Resource-bounded Baire category : a stronger approach. Proceedings of the Tenth Annual IEEE Conference on Structure in Complexity Theory, pages 182-192, 1995.

[9] S. A. Fenner. Gales and supergales are equivalent for defining constructive hausdorff dimension. Technical Report CC/0203017, Arxiv.org, August 2002.

[10] L. Fortnow and Jack Lutz. Prediction and dimension. Journal of Computer and System Sciences, 70:570-589, 2005.

[11] John M. Hitchcock. Small spans in scaled dimension. Proc. 19th ann. IEEE Conference on Computational Complexity, pages 104-112, 2004.

[12] D. Juedes and J. Lutz. The complexity and distribution of hard problems. Proceedings of the 34th FOCS Conference, pages 177-185, 1993.

[13] A. K. Lorentz and J. H. Lutz. Genericity and randomness over feasible probability measures. Theoretical Computer Science, 207:245-259, 1998.

[14] Jack H. Lutz. Effective fractal dimensions. Math. Log. Q., 51(1):62-72, 2005.

[15] J.H. Lutz. Category and measure in complexity classes. SIAM Journal on Computing, 19:1100-1131, 1990.

[16] J.H. Lutz. Almost everywhere high nonuniform complexity. Journal of Computer and System Sciences, 44:220-258, 1992.

[17] J.H. Lutz. The quantitative structure of exponential time. In L.A. Hemaspaandra and A.L. Selman, editors, Complexity Theory Retrospective II, pages 225-260. Springer, 1997.

[18] J.H. Lutz. Dimension in complexity classes. Proceedings of the 15th Annual IEEE Conference on Computational Complexity, pages 158-169, 2000. 
[19] J.H. Lutz. The dimensions of individual strings and sequences. Information and Computation, 187:49-79, 2003.

[20] P. Moser. Generic density and small span theorem. Proceedings of the 15th International Symposium on Fundamentals of Computation Theory, SpringerVerlag, pages 92-102, 2005.

[21] P. Moser. Baire categories on small complexity classes and meager-comeager laws. Accepted for publication in Information and Computation, 2007. 